\title{
Transfer Matrix Approach to One-Dimensional Electron Transport in Graphene Sheets with Piecewise Constant Potentials
}

\author{
S.R. GHAZANFARI* \\ Physics Department, Islamic Azad University Fars Science and Research Branch, P.O. Box 74715-181, Marvdasht, \\ Iran
}

(Received January 10, 2012; in final form November 4, 2012)

\begin{abstract}
We studied one-dimensional electron transport in a system composed of two monolayer graphene sheets with an optional arrangement of different constant rectangular electrostatic potential barriers between them. We derived a generalized transfer matrix for the electron which passes through this system. Finally, we examined our model by applying it on a well known rectangular shape constant potential barrier and we obtained the same result from our method similar to the others.
\end{abstract}

DOI: 10.12693/APhysPolA.123.148

PACS: 81.05.ue, 72.80.vp, 75.70.cn

\section{Introduction}

Graphene is a two-dimensional (2D) monolayer of carbon atoms that forms a planar hexagonal lattice which can be assumed as a one-atom thick sheet of carbon atoms, pulled out of graphite bulk by micro mechanical cleavage. Graphene was first produced by Geim's group in Manchester University in 2004 [1].

Because of its monolayer honeycomb lattice, electrons in graphene behave like massless Dirac fermions [2] and obey two-dimensional Dirac-like equation $[3,4]$. This is responsible for most unusual properties of graphene, for instance, emersion of the Shubnikov-de Haas (SdH) oscillations in graphene, by applying a time varying electric field instead of usual magnetic one [5]. An electrostatically created $n-p$ junction in monolayer graphene perfectly transmits those quasiparticles that approach it perpendicularly [6]. Due to the chiral nature of massless Dirac fermions in graphene, some relativistic experiments, like the Klein paradox [7] has been studied in condensed-matter by using an electrostatic barrier on a single layer graphene, theoretically [8] and experimentally [9]. In addition a remarkable property of graphene will be in heterostructures, for example a planar heterostructure composed of two graphene sheets and a narrow-gap semiconductor ribbon between them, can be applied as a gate [10].

In this letter we will find out a generalized transfer matrix for the electron transport through two monolayer graphene sheets with an optional arrangement of different constant rectangular electrostatic potential barriers between them. Electron behaves like massless Dirac fermions in graphene regions and it can be massive or massless in potential region (region between the graphenes). If the electron behaves like massless Dirac particles in each place, transmission probability will be unity in one-dimensional motion [8]. Here we are going to

*corresponding author; e-mail: Sr.Ghazanfari@yahoo.com calculate the transfer matrix of the system when electron is massive in the potential region. Obtaining the transfer matrix will let us to use it for calculating the transmission probability. Finally, we will examine our model by applying it on a well known system, when the potential region is a constant rectangular electrostatic potential barrier, on a single layer graphene.

The paper is organized as follows. In Sect. 2 we present the plane wave solutions of massless and massive Dirac equations. In Sect. 3 we will derive the transfer matrix and finally will use it in Sect. 4 .

\section{Electron in different regions}

In the hexagonal reciprocal lattice of graphene there are two non-equivalent points $\boldsymbol{K}, \boldsymbol{K}^{\prime}$, the so called Dirac points, at the corners of the first Brillouin zone [11, 12]. Around each of the Dirac points, low energy electrons and holes have linear dispersion

$$
E(\boldsymbol{K})= \pm \hbar v_{\mathrm{F}}|\boldsymbol{K}|,
$$

with two-dimensional wave vector $K$ and the Fermi velocity $v_{\mathrm{F}} \approx 10^{6} \mathrm{~m} / \mathrm{s}[11,12]$. Thus the conduction (electrons) and valence (holes) conical bands touch at the points $\boldsymbol{K}, \boldsymbol{K}^{\prime}(E(\boldsymbol{K})=0)$ producing two nonequivalent valleys. This makes graphene a gapless semiconductor with relativistic-like dispersion of the excitations [13].

An electron in graphene lattice is described by a four-component spinor $\left(\psi_{A}^{+}, \psi_{B}^{-}, \psi_{A}^{+}, \psi_{B}^{-}\right)$in which, $\psi_{A(B)}^{+}$is referred to the amplitude of the electron wave function on sublattice $A(B)$ of the hexagonal structure with wave vector centered around the valley $\boldsymbol{K}$ and $\psi_{A(B)}^{-}$is the corresponding wave function around the valley $\boldsymbol{K}^{\prime}$ [13].

When we assume the graphene in $x-y$ plane, this four-component spinor satisfies the Dirac equation of the form [13]:

$$
\begin{aligned}
& -\mathrm{i} v_{\mathrm{F}}\left(\begin{array}{cc}
H^{+} & 0 \\
0 & H^{-}
\end{array}\right) \psi=E \psi, \\
& H^{ \pm}=\sigma_{x} \partial_{x} \pm \sigma_{y} \partial_{y},
\end{aligned}
$$

where $\sigma_{x}$ and $\sigma_{y}$ are the Pauli matrices describing pseudo 
spin space of two sublattices $A$ and $B$ (hereafter $\hbar=1$ ). Electrons in graphene obey two-dimensional massless Dirac like Hamiltonian around the points $\boldsymbol{K}$ and $\boldsymbol{K}^{\prime}$ as

$$
H=v_{\mathrm{F}} \boldsymbol{\sigma} \cdot \boldsymbol{K},
$$

for $\boldsymbol{K}$ points and

$$
H=v_{\mathrm{F}} \boldsymbol{\sigma} \cdot \boldsymbol{K}^{\prime}
$$

for $\boldsymbol{K}^{\prime}$, where $\boldsymbol{K}=\left(k_{x}, k_{y}\right), \boldsymbol{K}^{\prime}=\left(k_{x},-k_{y}\right), k_{x, y}=$ $-\mathrm{i} \partial_{x, y}$ and $\boldsymbol{\sigma}=\left(\sigma_{x}, \sigma_{y}\right)$.

Since every permutation of the Pauli matrices in two-dimensional Dirac equation is allowed [14], we can choose the $\boldsymbol{\sigma}$ matrices in Eqs. (4), (5) as $\boldsymbol{\sigma}=\left(\sigma_{z}, \sigma_{y}\right)$ and assume the graphene sheet in the $z-y$ plane. Now we have $\boldsymbol{K}=\left(k_{z}, k_{y}\right), \boldsymbol{K}^{\prime}=\left(k_{z},-k_{y}\right)$ and $k_{z, y}=-\mathrm{i} \partial_{z, y}$ and Hamiltonian of graphene will be in the form below

$$
H=v_{\mathrm{F}}\left(\begin{array}{cc}
\boldsymbol{\sigma} \cdot \boldsymbol{K} & 0 \\
0 & \boldsymbol{\sigma} \cdot \boldsymbol{K}^{\prime}
\end{array}\right) .
$$

Performing two unitary transformations $U_{1}=\left(\begin{array}{cc}I & 0 \\ 0 & \sigma_{y}\end{array}\right)$ and $U_{2}=\frac{1}{\sqrt{2}}\left(\begin{array}{cc}I & I \\ I & -I\end{array}\right)(I$ is $2 \times 2$ unit matrix $)$ sequentially to graphene's Hamiltonian [15], will lead us to obtain the Dirac-like Hamiltonian as

$$
H_{\mathrm{D}}=v_{\mathrm{F}} \alpha K \text {, }
$$

where $\alpha=\left(\begin{array}{cc}0 & \boldsymbol{\sigma} \\ \boldsymbol{\sigma} & 0\end{array}\right)$ are the $\alpha$ Dirac matrices.

Now we have a two-dimensional massless Dirac equation in $z-y$ plane. For energy $E(E>0)$ and helicity $\lambda$, the wave equation along different directions of the $z$ axis will be [16]:

$$
\psi_{ \pm}(Z)=\frac{1}{\sqrt{2}}\left(\begin{array}{c}
\xi(\lambda) \\
\pm \lambda \xi(\lambda)
\end{array}\right) \mathrm{e}^{-\mathrm{i} E t \pm \mathrm{i} \boldsymbol{K} \cdot \boldsymbol{Z}}
$$

where $\boldsymbol{K}$ is the momentum, $\xi(\lambda)$ is the normalized Pauli spinor of the helicity basis, $\psi_{+}(Z)$ is the wave equation along positive direction of the axis and $\psi_{-}(Z)$ is the wave equation along negative one.

So far we calculated the wave equations in graphene regions, now we are going to find the wave equations in potential region. We will assume the potential region in a space domain $D$, and apply an external electromagnetic field on this domain for creation of the potential barrier. The Dirac equation for the motion of an electron with charge $-e$ and the mass $m$, in an external electromagnetic field $A_{\mu}$ is

$$
\gamma^{\mu}\left(\mathrm{i} \partial_{\mu}-e A_{\mu}\right) \psi-m \psi=0 \text {. }
$$

And here we choosed the $\gamma$-matrices in standard form [17]. By arranging the $\boldsymbol{A}(x)=0$ and $e A_{0}(x)=$ $V=$ const for any $\boldsymbol{x} \in D$ the Dirac equation can be analytically solved. So with momentum $\boldsymbol{K}, E(K)=$ $\sqrt{m^{2}+\boldsymbol{K}^{2}}+V$ and helicity $\lambda$ in the standard normalization we have $[17,18]$ :

$$
\begin{aligned}
& \psi_{E, \lambda}(X)=\frac{1}{\sqrt{2 m}}\left(\begin{array}{c}
\sqrt{E(K)-V+m} \xi(\lambda) \\
\lambda \sqrt{E(K)-V-m} \xi(\lambda)
\end{array}\right) \\
& \times \mathrm{e}^{-\mathrm{i} E t+\mathrm{i} K X} .
\end{aligned}
$$

This is a general solution for Eq. (9) with positive energy $E$ and it is held only for $E \geq V+m$. To obtain one-dimensional solution, we can orient the momentum $\boldsymbol{K}$ along to a fixed direction, in this case we will orient it along the $z$ axis.

We assumed that sigma matrices in (3)-(7) represent pseudospin (due to sublattices $A$ and $B$ in graphene) in Dirac-like equation for graphene regions and sigma matrices in (9) represent the real spin of electron in narrow-gap semiconductor region. Pseudo spin is not related to the real physical spin and sigma matrices in graphene and potential regions provide the spinor shape of wave functions. This difference is not going to affect our calculations, because in narrow-gap semiconductor Hamiltonian (9) we are not considering the effect of real spin (we are looking for electrostatic barriers effect only).

Since the spin is projected on the $z$ axis, the helicity basis spinors are equal to spins basis one. It means that two-component spinor $\xi(\lambda)$ is in the form of $\xi(1)=(1,0)^{\mathrm{T}}$ and $\xi(-1)=(0,1)^{\mathrm{T}}$. Now we will set an arbitrary arrangement of rectangular constant potentials between two sheets of graphene. We can assume $N$ rectangular constant potential barriers whereas each of them has optional width and height as illustrated in Fig. 1.

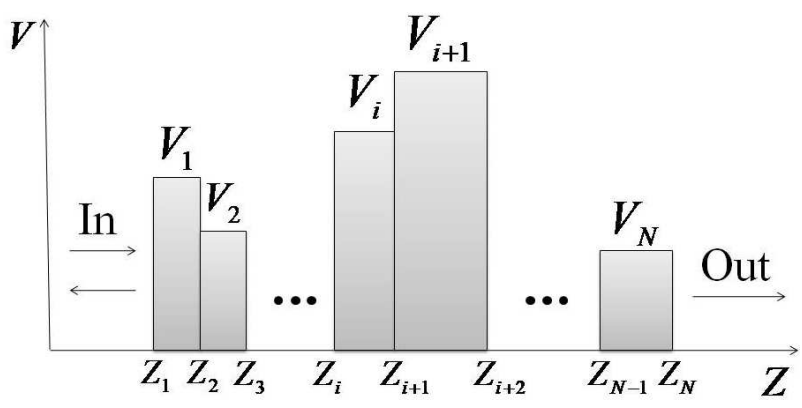

Fig. 1. Arbitrary arrangement of constant rectangular barriers.

These potentials will be in specified intervals denoted by $D_{i}=\left[z_{i}, z_{i+1}\right]$ and each interval has a specified constant experimental potential, $V_{i}$. Two graphene sheets will be in two intervals $D_{\text {in }} \equiv D_{0}=\left(-\infty, z_{1}\right]$ and $D_{\text {out }} \equiv D_{N+1}=\left[z_{N}, \infty\right)$, and there is no potential in these regions, $V_{0}=V_{N+1}=0$. Here we will assume that electron in each interval $D_{i}$ has an effective mass $m_{i}$ and relativistic potential $\hat{V}_{i}=V_{i}+\delta m_{i}$ instead of experimental one, where $\delta m_{i}=m-m_{i}$ [19]. In each interval there is a plane wave solution with energy $E$ and helicity $\lambda$ along the positive direction of the $z$ axis. From Eq. (10) we have 


$$
\phi_{E, \lambda}^{i}(t, z)=\frac{1}{\sqrt{2}}\left(\begin{array}{c}
k_{i}^{(+)} \xi(\lambda) \\
\lambda k_{i}^{(-)} \xi(\lambda)
\end{array}\right) \mathrm{e}^{-\mathrm{i} E t+\mathrm{i} k_{i} z},
$$

where for graphene regions $k_{0}^{ \pm}=k_{N+1}^{ \pm}=1$ and for the potential region $k_{i}^{( \pm)}=\sqrt{\frac{E-\hat{V}_{i} \pm m_{i}}{m_{i}}}$. The scalar momentum for graphene regions is $\boldsymbol{K}_{0}=\boldsymbol{K}_{N+1}=\frac{E}{v_{\mathrm{F}}}$ and for the rest of the system the momentum is $\boldsymbol{K}_{i} \stackrel{{ }_{\mathrm{F}}}{=}$ $\sqrt{\left(E-\hat{V}_{i}\right)^{2}-m_{i}^{2}}$.

We have plane wave solutions with the same $E$ and helicity $\lambda$ but propagating in another direction of the $z$ axis as

$$
\chi_{E, \lambda}^{i}(t, z)=\frac{1}{\sqrt{2}}\left(\begin{array}{c}
k_{i}^{(+)} \xi(\lambda) \\
-\lambda k_{i}^{(-)} \xi(\lambda)
\end{array}\right) \mathrm{e}^{-\mathrm{i} E t-\mathrm{i} K_{i} z},
$$

where $k_{i}^{ \pm}$and $K_{i}$ in this equation are the same as the parameters used in Eq. (11). The general plane wave solutions with energy $E$ and helicity $\lambda$ in each domain $D_{i}$ is a linear combination of $\phi^{i}$ and $\chi^{i}$, which is

$$
\Psi_{E, \lambda}^{i}(t, z)=A_{i} \phi_{E, \lambda}^{i}(t, z)+B_{i} \chi_{E, \lambda}^{i}(t, z) .
$$

Here $A_{i}$ and $B_{i}$ are arbitrary complex numbers.

\section{Transfer matrix}

Now we are going to find the transfer matrix of electron transport through the defined system. Energy satisfies the condition $E \geq V+m$ in entire of the system and all of the solutions $(11,12)$ have the identical fixed energy $E$ and helicity $\lambda$ in each interval. When the number of intervals $(N)$ is large, we can use the associated two-dimensional vectors, instead of Eq. (13) [20]:

$$
v_{i}(z)=\left(\begin{array}{c}
A_{i} \mathrm{e}^{\mathrm{i} K_{i} z} \\
B_{i} \mathrm{e}^{-\mathrm{i} K_{i} z}
\end{array}\right) .
$$

These vectors represent incoming and outgoing wave functions in each interval. Thus the vectors (14) become the basic elements of the transfer matrix for rectangular barrier, and all we have to do is to find the transfer matrix $M$ which relates the initial and final vectors as

$$
v_{0}\left(z_{1}\right)=M v_{N+1}\left(z_{N+1}\right) .
$$

The wave Eq. (13) is continuous in each point $z_{i}$, and from boundary condition we have

$$
\Psi_{E, \lambda}^{i-1}\left(t, z_{i}\right)=\Psi_{E, \lambda}^{i}\left(t, z_{i}\right)
$$

and $i=1,2, \ldots, N+1$. By applying some calculation we can reach to a simple relation among the associated vectors

$$
v_{i-1}\left(z_{i}\right)=M_{i} v_{i}\left(z_{i}\right),
$$

where $M_{i}$ matrices are

$$
M_{i}=\frac{1}{2}\left(\begin{array}{cc}
r_{i}^{+}+r_{i}^{-} & r_{i}^{+}-r_{i}^{-} \\
r_{i}^{+}-r_{i}^{-} & r_{i}^{+}+r_{i}^{-}
\end{array}\right)
$$

and

$$
r_{i}^{+}=\frac{k_{i}^{(+)}}{k_{i-1}^{(+)}}, \quad r_{i}^{-}=\frac{k_{i}^{(-)}}{k_{i-1}^{(-)}} .
$$

At last we have the translation matrices as

$$
T_{i}=\left(\begin{array}{cc}
\mathrm{e}^{-\mathrm{i} K_{i}\left(z_{i+1}-z_{i}\right)} & 0 \\
0 & \mathrm{e}^{\mathrm{i} K_{i}\left(z_{i+1}-z_{i}\right)}
\end{array}\right),
$$

that transforms $v_{i}\left(z_{i+1}\right)$ into $v_{i}\left(z_{i}\right)=T_{i} v_{i}\left(z_{i+1}\right)$. Now we can introduce the general formula for the transfer matrix as

$$
M=\left[\prod_{i=1}^{N} M_{i} T_{i}\right] M_{N+1} .
$$

This matrix relates the initial and final vectors through an arbitrary set of constant rectangular potential barriers.

\section{Constant potential}

In this section we will examine our model by applying it on the system, in which we have a rectangular shape potential barrier infinite along the $y$-axis on a monolayer graphene, as the potential region

$$
V(z)=\left\{\begin{array}{cl}
V, & 0<z<d \\
0 & \text { otherwise }
\end{array}\right.
$$

The general scheme of this system is shown in Fig. 2.

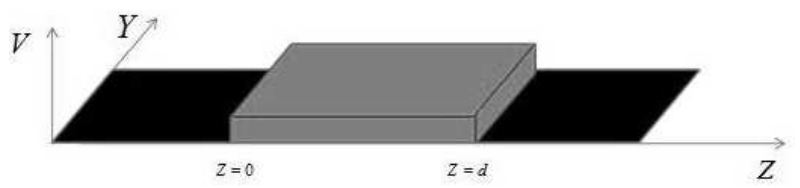

Fig. 2. A constant rectangular potential barrier between two graphene sheets.

Here electron obeys the massless Dirac-like equation, entire of the system. From Eq. (8) we have the wave function for electron in graphene regions with zero and nonzero potential, in both directions of the $z$ axis. In this case we have $k_{0}^{ \pm}=k_{N+1}^{ \pm}=k_{i}^{ \pm}=1$ and the momentum in the potential region is $\boldsymbol{K}_{i}=\frac{E-V}{v_{\mathrm{F}}}$. We can assume the number of rectangular barriers as $N=1$, because the potential value does not change in the interval $0<z<d$. After calculating $r_{i}^{ \pm}$the $M_{i}$ matrix will be

$$
M_{1}=\left(\begin{array}{ll}
1 & 0 \\
0 & 1
\end{array}\right) .
$$

For the transmission matrix we have

$$
T_{1}=\left(\begin{array}{cc}
\mathrm{e}^{-\mathrm{i} \frac{E-V}{v_{F}} d} & 0 \\
0 & \mathrm{e}^{\mathrm{i} \frac{E-V}{v_{\mathrm{F}}} d}
\end{array}\right)
$$

and $M_{N+1=2}$ is

$$
M_{2}=\left(\begin{array}{ll}
1 & 0 \\
0 & 1
\end{array}\right) .
$$

Finally the transfer matrix from Eq. (24) will be

$$
M=\left(\begin{array}{cc}
\mathrm{e}^{-\mathrm{i} \frac{E-V}{v_{\mathrm{F}}} d} & 0 \\
0 & \mathrm{e}^{\mathrm{i} \frac{E-V}{v_{\mathrm{F}}} d}
\end{array}\right) .
$$

The transmission coefficient is 


$$
\mathcal{T}=\frac{\left|A_{N+1=2}\right|^{2}}{\left|A_{0}\right|^{2}}=\left|M_{11}\right|^{-2} .
$$

Obviously it is equal to unity $(\mathcal{T}=1)$, and it is in correspondence with Ref. [8].

\section{Conclusions}

In this paper, we have studied the one-dimensional electron transport through a system consisting of an arbitrary set of constant rectangular electrostatic potential barriers, between two graphene sheets. We introduced a generalized transfer matrix for this system where the electron in potential region behaves like a massive particle. Finally, we tested our model by calculating the electron transmission probability for a rectangular constant electrostatic potential barrier on a single layer graphene and its result was the same as the others.

\section{References}

[1] K.S. Novoselov, A.K. Geim, S.V. Morozov, D. Jiang, Y. Zhang, S.V. Dubonos, I.V. Grigorieva, A.A. Firsov, Science 306, 666 (2004)

[2] D. DiVincenzo, E. Mele, Phys. Rev. B 29, 1685 (1984)

[3] A.H. Castro Neto, F. Guinea, N.M.R. Reres, K.S. Novoselov, A.K. Geim, Rev. Mod. Phys. 81, 109 (2009)

[4] A. Rycerz, J. Tworzydlo, C.W.J. Beenakker, Nat. Phys. 3, 172 (2007)

[5] V.A. Yampol'skii, S. Savel'ev, F. Nori, New J. Phys. 10, 053024 (2008)
[6] V.V. Cheianov, V.I. Falko, Phys. Rev. B 74, 041403 (2006)

[7] O. Klein, Z. Phys. 53, 157 (1929).

[8] M.I. Katsnelson, K.S. Novoselov, A.K. Geim, Nat. Phys. 2, 620 (2006)

[9] N. Stander, B. Huard, D. Goldhaber-Gordon, Phys. Rev. Lett. 102, 026807 (2009)

[10] P.V. Ratnikov, A.P. Silin, Bull. Lebedev Phys. Inst. 35, 328 (2008)

[11] K.S. Novoselov, A.K. Geim, S.V. Morozov, D. Jiang, M.I. Katsnelson, I.V. Grigorieva, S.V. Dubonos, A.A. Firsov, Nature 438, 197 (2005).

[12] Y. Zhang, Y.-W. Tan, H.L. Stormer, P. Kim, Nature 438, 201 (2005)

[13] P.R. Wallace, Phys. Rev. 71, 622 (1947).

[14] J.N. Ginocchio, A. Leviatan, J. Meng, S.-G. Zhou, Phys. Rev. C 69, 034303 (2004)

[15] P.V. Ratnikov, Pisma Zh. Eksp. Teor. Fiz. 87, 343 (2008) [ JETP Lett. 87, 292 (2008)].

[16] S.S. Schweber, An Introduction to Relativistic Quantum Field Theory, Harper and Row, New York 1962.

[17] B. Thaller, The Dirac Equation, Springer-Verlag, Berlin 1992

[18] W.G. Bagrov, D.M. Gitman, Exact Solutions of Relativistic Wave Equations, Kluwer Academic, Dordrecht 1990.

[19] I.I. Cotaescu, P. Gravila, M. Paulescu, Phys. Lett. A 366, 363 (2007)

[20] R. Renan, M.H. Pacheco, C.A.S. Almeida, J. Phys. A, Math. Gen. 33, L509 (2000). 